\title{
Linx
}

Revue des linguistes de l'université Paris X Nanterre

$11 \mid 1999$

Typologie des langues, universaux linguistiques

\section{Pour une typologie sémantique}

\section{Bernard Pottier}

\section{Q OpenEdition \\ Journals}

Édition électronique

URL : http://journals.openedition.org/linx/856

DOI : 10.4000/linx.856

ISSN : 2118-9692

\section{Éditeur}

Presses universitaires de Paris Nanterre

\section{Édition imprimée}

Date de publication : 1 décembre 1999

Pagination : 11-14

ISSN : 0246-8743

\section{Référence électronique}

Bernard Pottier, « Pour une typologie sémantique », Linx [En ligne], 11 | 1999, mis en ligne le 29 juin 2012, consulté le 19 avril 2019. URL : http://journals.openedition.org/linx/856; DOI : 10.4000/linx.856

Ce document a été généré automatiquement le 19 avril 2019

Département de Sciences du langage, Université Paris Ouest 


\title{
Pour une typologie sémantique
}

\author{
Bernard Pottier
}

1 Les études de caractère typologique peuvent s'appliquer à tous les domaines de la linguistique. C'est en morphosyntaxe qu'elles ont connu et connaissent le plus de succès, et on le comprend, étant donné que la "grammaire» constitue l'armature des réalisations discursives. Les domaines phonique ou textuel sont également abordés, dans une moindre mesure.

2 Même si l'on ne conçoit pas une morphosyntaxe qui négligerait la sémantique, celle-ci est rarement considérée pour elle-même. L'aspect, la détermination, la quantification, la subordination, etc., sont envisagés en fonction de leurs manifestations dans les langues, ce qui est tout naturel. Aussi se dirige-t-on vers des généralisations qui, si on accepte un saut qualitatif, conduisent à poser certains universaux. Ceux-ci, plutôt que des concepts abstraits, sont essentiellement des mécanismes ordonnéset dynamiques, dont nous avons souvent parlé1.

3 Notre but, aujourd'hui, est de suggérer de s'intéresser à une typologie sémantique, ce qui suppose que les phénomènes envisagés sont vus indépendamment des catégories syntaxiques qui les enfermeront dans une langue particulière. On peut poser une typologie sémantique universelle et une typologie sémantique particulière. C'est cette dernière qui est prise en compte dans les études traditionnelles de typologie.

4 Donnons quelques exemples de sémantique universelle. D'abord le cas le plus connu, celui des tropes : la métaphore et la métonymie existent partout et elles sont valables pour tous types de signes linguistiques : elles sont transcatégorielles.

5 Nous posons également le caractère universel des aires d'instanciation des représentations mentales. Ainsi le lexème 'arriv-' peut-il être saisi dans une aire existentielle ( il arrive que $\mathrm{P} »)$, spatiale ( Jean arrive à la maison»), modale ( Jean arrive à fermer sa valise », cf. pouvoir), de la même façon que 'sur' peut être saisi à travers l'espace («sur la table»), le temps («le surlendemain»), le notionnel ( «interroger sur les origines de la maladie »). On peut retenir les cinq aires qui couvrent les valeurs combinatoires: existence, espace, temps, notion, modalité. Il s'agit d' homologies en parallèle et non de métaphore à partir d'une aire particulière (comme celle de l'espace, chère aux « localistes »). 
Lorsque Ch. de Lamberterie ${ }^{2}$ a étudié l'étymologie commune à l'arménien erku et au latin duo, avec le sens de "deux», il a cité de nombreux dérivés dont les significations semblent s'éloigner fortement du sens de départ : «loin », « douleur », « douter », etc... Nous proposons d'y voir une représentation mentale de dualité, par rapport à une unité d'origine, d'où les notions de "séparation ", « distanciation ", « déchirement ", « mise en parallèle de deux éléments " («doute», «alternative», etc.), ce qui peut se figurer comme :

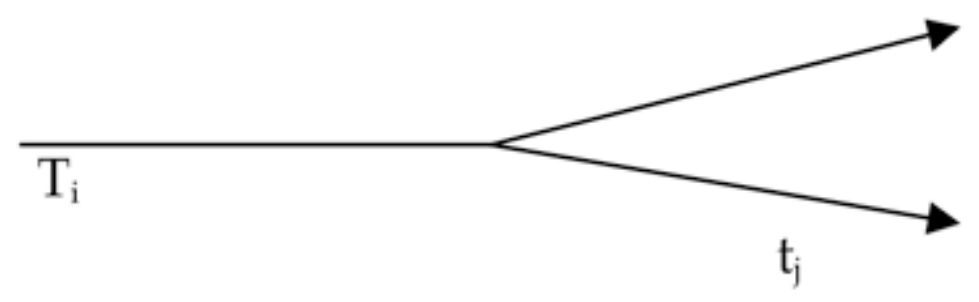

7 On connaît les distinctions du latin concernant les «postures»: stare («la statue se dressait à Delphes »), sedere («le siège d'une ville»), iacere («maison en contrebas», " être dans la douleur»), pendere (" être en vente", "se dit aussi des nuages»). Ces contextes montrent comment le sème d'orientation contenu dans ces verbes est en harmonie ( «isosémie ») avec les visions événementielles retenues par le locuteur. Il est curieux de constater qu'on retrouve les mêmes phénomènes dans une langue amérindienne de Colombie, le sikwani, étudié par Fr. Queixalós ${ }^{3}$. Les quatre verbes de posture $n u, e, b o, r u$, servent de présentateurs de classes de visions nominales (c'est-à-dire d'entités), et on a respectivement le «mur» (que l'on dresse), la «maison» (qui a une assise), la « forêt », la « dalle » (qui git sur la terre) ou les « nuages » (qui sont suspendus au-dessus de nos têtes). Voilà donc quatre attitudes d'expérience de l'homme qui ont leur parallèle dans des classifications linguistiques exprimées tantôt par une combinatoire dominante, tantôt par une morphologie explicite, et cela dans des cultures très variées.

Un dernier exemple. L'affectation d'une qualité à une entité est fréquemment différenciée selon le degré d'intimité lié à la personne et à la vision du locuteur. "L'inhérence /v/ la relativité » motive le choix des deux verbes d'attribution en irlandais, en espagnol, ou en portugais, même si le seuil qui fait passer de l'un à l'autre ne se situe pas au même endroit sur le continuum qui sous-tend la distinction (is/ta, ser/estar ). En japonais, l'attribution d'une propriété modale par nature est immédiate si la première personne en est l'auteur (litt. « je suis triste »), et médiate dans le cas contraire (litt. « tu me sembles triste »). Le français dira: « je suis triste / je te trouve triste » par exemple (cf. mon ancienne proposition de justifier la deuxième personne du singulier du présent espagnol de «ser" issue de la forme du futur latin eris > eres, par cette même attitude modale $)^{4}$. Les langues sont sensibles à cette distinction qui correspond à des visions différentes de l'expérience, mais chacune d'elles a ses propres solutions, plus ou moins grammaticalisées.

9 Les conditions de l'expérience du monde conduisent à des parcours mentaux du même ordre. A partir de là, les conditions socioculturelles amènent les langues à intégrer plus ou moins ces distinctions dans leur morphosyntaxe.

10 Une typologie sémantique universelle peut être une hypothèse utile au moment de caractériser les spécificités des langues. 


\section{BIBLIOGRAPHIE}

PotTIER, B. (1994) «Les schèmes mentaux et la langue », Modèles linguistiques, P.U. Lille, XV-2, pp.7-50 .

DE LAMBERTERIE, C. (à paraître en 2000) Communication du 23-10-1998 faite à l'Académie des Inscriptions et Belles-Lettres.

QueIXALós, F. (1998) Nom, verbe et prédicat en sikuani, Paris-Louvain, Peeters.

POTTIER, B. (1977) « La forma 'eres' », in Estudios ofrecidos a Emilio Alarcos Llorach, Oviedo, t.1, pp. 207-208.

\section{NOTES}

1. B. Pottier, «Les schèmes mentaux et la langue ». Modèles linguistiques, P.U. Lille, 1994, XV-2, pp. 7-50.

2. Ch. de Lamberterie, Communication du 23.10.1998 faite à l'Académie des Inscriptions et bellesLettres (à paraître en 2000).

3. Fr. Queixalós, F. (1998) Nom, verbe et prédicat en sikuani, Paris-Louvain, Peeters.

4. B. Pottier, « La forma 'eres' » in Estudios ofrecidos a Emilio Alarcos Llorach, Oviedo, 1977, t. 1, pp. 207-208.

\section{AUTEUR}

BERNARD POTTIER

Université Paris IV 